\title{
Large retention cyst with chondromatous metaplasia in the esophagus
}

A 52-year-old man with a 2-year history of diabetes mellitus underwent esophagogastroduodenoscopy (EGD) as part of a medical check-up. He had no symptoms. EGD revealed a semipedunculated polyp, $50 \mathrm{~mm}$ long and $20 \mathrm{~mm}$ in diameter, in the thoracic esophagus ( $\bullet$ Fig. 1a). The polyp was covered by normal mucosa with mildly dilated vessels ( $\bullet$ Fig. $\mathbf{1}$ b,c). Endoscopic ultrasound (EUS) showed multilocular hypoechoic areas within the second layer ( Fig. 2) and an intact fourth layer in the stalk of the polyp. Double-contrast esophagogram also showed a semipedunculated polyp hanging down from the thoracic esophagus ( Fig. 3). Although the esophageal polyp was thought likely to be a submucosal cyst of the esophagus, the patient requested treatment of the polyp before symptoms, such as discomfort in the esophagus or dysphagia, developed. The patient understood the risks of endoscopic treatment and provided written informed consent.

Endoscopic treatment was performed without any complications. The size of the resected specimen was $53 \times 23 \times 15 \mathrm{~mm}$ ( $\bullet$ Fig. 4). Histopathological evaluation of the resected specimen demonstrated multiple cysts lined internally by cuboidal epithelium in the submucosa and a small chondrosis in the stroma, and that the mucosa covering the polyp consisted of normal squamous epithelium ( $\bullet$ Fig.5). The polyp was diagnosed as a retention cyst with chondromatous metaplasia in the esophagus.

Although retention cysts are the most common benign lesion in the pharynx [1], they are uncommon in the esophagus [2-4]. An esophageal squamous cell carcinoma coexisting with a submucosal retention cyst has been reported [4]. To our knowledge, this is the first report of a large esophageal retention cyst with chondromatous metaplasia that was treated endoscopically. As this cyst did not consist of ciliated epithelium, it is assumed that it was not of bronchial origin. We were not able to determine the cause of the chondrosis.

Endoscopy_UCTN_Code_CCL_1AB_2AC_3AB
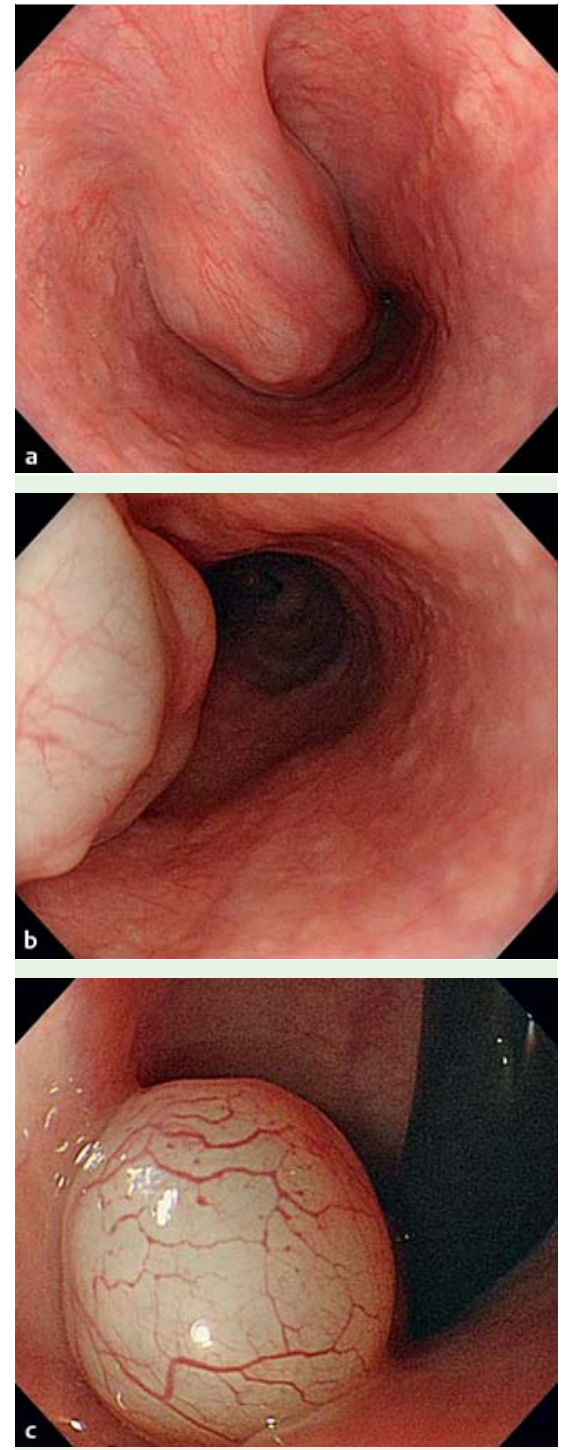

Fig. 1 Endoscopic views within the thoracic esophagus showing: a a semipedunculated polyp; $\mathbf{b}$ normal appearance of the mucosa covering the polyp; $\mathbf{c}$ following a retroflexion maneuver, the head of the polyp covered with normal mucosa and mildly dilated vessels.

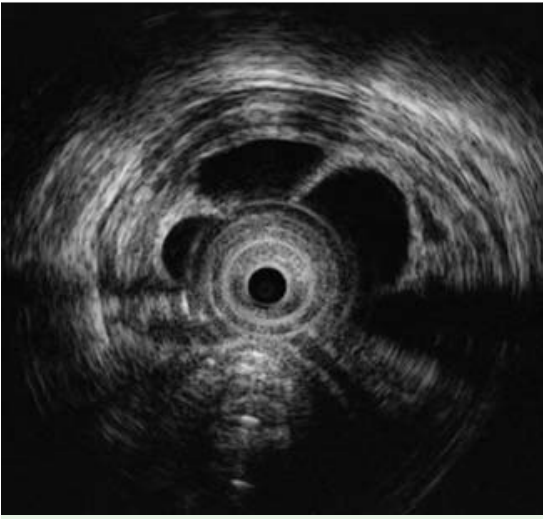

Fig. 2 Endoscopic ultrasound (EUS) demonstrating multilocular hypoechoic areas within the second layer of the polyp.

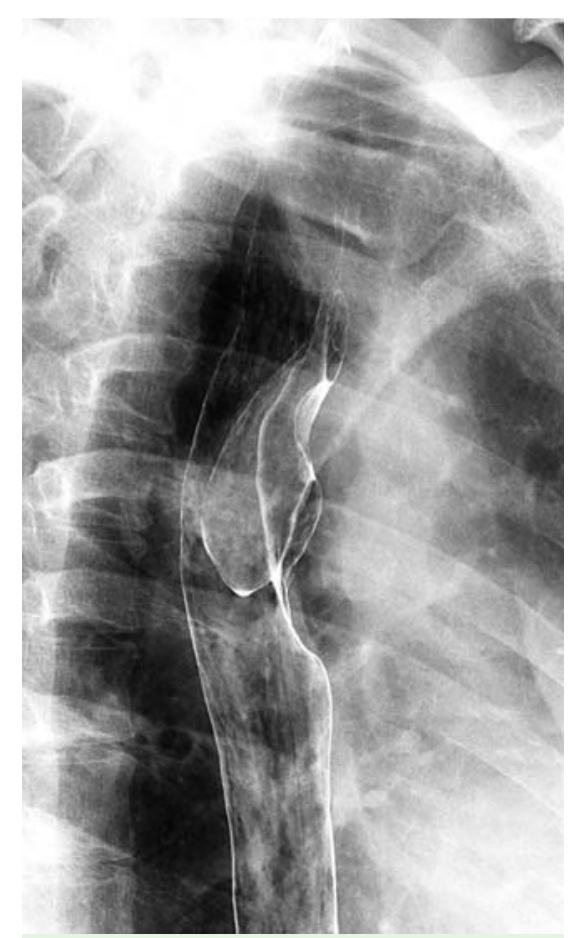

Fig. 3 Double-contrast esophagogram showing the semipedunculated polyp in the thoracic esophagus. 


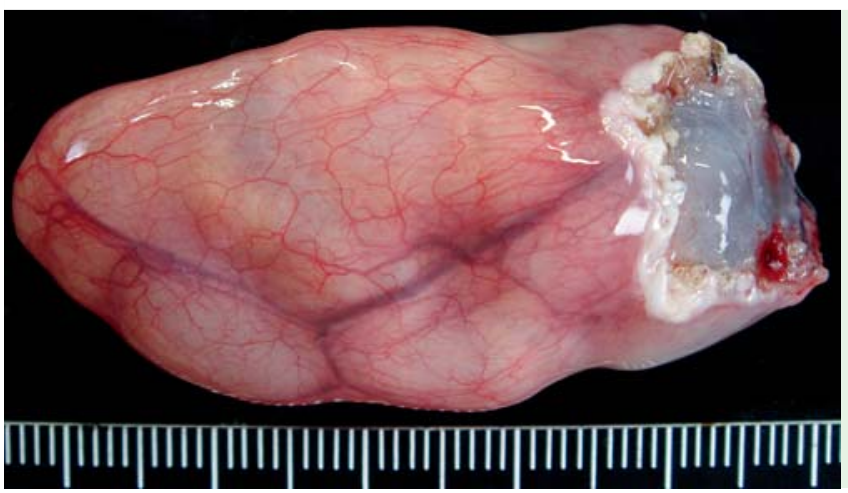

Fig. 4 Macroscopic appearance of the resected polyp, $53 \times$ $23 \times 15 \mathrm{~mm}$ in size, showing normal surface mucosa.
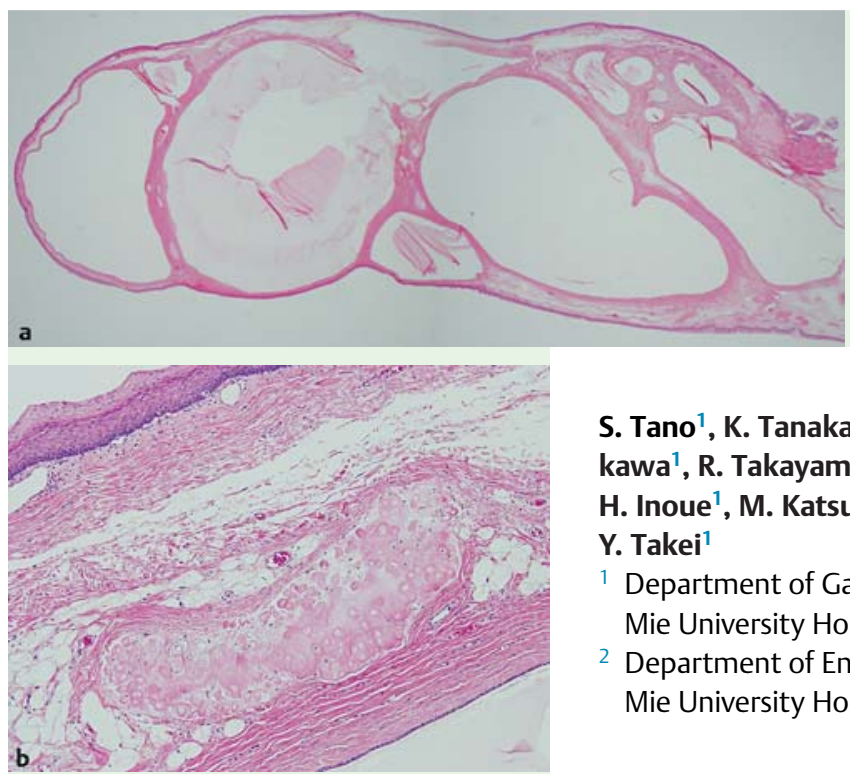

S. Tano ${ }^{1}$, K. Tanaka ${ }^{2}$, T. Iguchi ${ }^{1}$, K. Nishikawa ${ }^{1}$, R. Takayama ${ }^{1}$, K. Ninomiya ${ }^{1}$, H. Inoue ${ }^{1}$, M. Katsurahara ${ }^{2}$, N. Horiki², Y. Takei ${ }^{1}$

1 Department of Gastroenterology, Mie University Hospital, Tsu, Japan

2 Department of Endoscopic Medicine, Mie University Hospital, Tsu, Japan

\section{References}

1 Woodfield CA, Levine MS, Rubesin SE et al. Pharyngeal retention cysts: radiographic findings in seven patients. AJR Am J Roentgenol 2005; 184: 793 - 796

2 Edgin R, Mekhjian HS. Esophageal retention cyst: unusual cause for dysphagia. J Clin Gastroenterol 1981; 3 Suppl 1: 57-59

3 Hover AR, Brady CE 3rd, Williams JR et al. Multiple retention cysts of the lower esophagus. J Clin Gastroenterol 1982; 4: 209-212

4 Toyoda H, Kobayashi N, Mukai K et al. Endoscopic resection of coexisting superficial carcinoma and a retention cyst. Endoscopy 2000; 32: S41

\section{Bibliography}

DOI $10.1055 / \mathrm{s}-0030-1256531$

Endoscopy 2011; 43: E262 -E263

(c) Georg Thieme Verlag KG Stuttgart · New York .

ISSN 0013-726X

\section{Corresponding author}

\section{K. Tanaka, MD}

Department of Endoscopic Medicine

Mie University Hospital

2-174 Edobashi, Tsu

Mie, 514-8507

Japan

Fax: +81-59-2315200

kyosuket@qa2.so-net.ne.jp
Fig. 5 Histological appearance of the polyp stained with hematoxylin and eosin (H\&E) showing: a that it consisted of multilocular cysts with a covering of normal squamous mucosa (magnification $\times 12.5$ ). $\mathbf{b}$ multiple cysts lined by cuboidal epithelium in the submucosa and a small chondrosis in the stroma (magnification $\times 200$ ). 